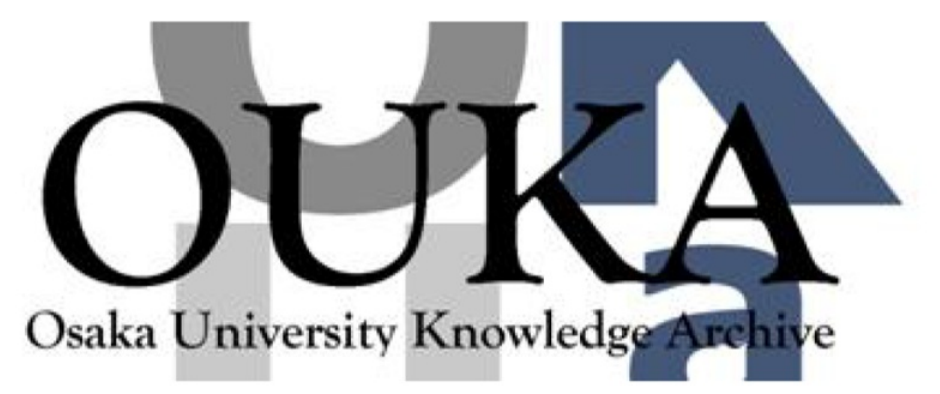

\begin{tabular}{|c|l|}
\hline Title & $\begin{array}{l}\text { Molecular dynamics analysis of the velocity } \\
\text { slip of a water and methanol Liquid mixture }\end{array}$ \\
\hline Author(s) & $\begin{array}{l}\text { Nakaoka, Satoshi; Yamaguchi, Yasutaka; Omori, } \\
\text { Takeshi et al. }\end{array}$ \\
\hline Citation & Physical Review E. 92(2) p. 022402 \\
\hline Issue Date & $2015-08-04$ \\
\hline oaire:version & VoR \\
\hline URL & https://hdl. handle. net/11094/82374 \\
\hline rights & Copyright 2015 by the American Physical Society \\
\hline Note & \\
\hline
\end{tabular}

Osaka University Knowledge Archive : OUKA

https://ir. Library. osaka-u. ac. jp/

Osaka University 


\title{
Molecular dynamics analysis of the velocity slip of a water and methanol liquid mixture
}

\author{
Satoshi Nakaoka, ${ }^{*}$ Yasutaka Yamaguchi, ${ }^{\dagger}$ and Takeshi Omori \\ Department of Mechanical Engineering, Osaka University and 2-1 Yamadaoka 565-0871, Suita, Japan \\ Masaru Kagawa, Tadashi Nakajima, and Hideo Fujimura \\ R\&D Center, Dai Nippon Printing Co., Ltd. and 1-1-3 Midorigahara 300-2646, Tsukuba, Japan \\ (Received 17 April 2015; revised manuscript received 29 June 2015; published 4 August 2015)
}

\begin{abstract}
The effect of methanol mixing on a nanoscale water flow was examined by using nonequilibrium molecular dynamics simulations of a Couette-type flow between nonpolarized smooth solid surfaces. Water and methanol molecules were uniformly mixed in the bulk, whereas at the solid-liquid interface methanol molecules showed a tendency to be adsorbed on the solid surface. Similar to a macroscale Couette flow, the shear stress exerted on the solid surface was equal to the shear stress in the liquid, showing that the mechanical balance holds in nanoscale. In addition, the shear stress in the liquid bulk was equal to the viscous stress which is a product of viscosity and velocity gradient. When more methanol molecules were adsorbed on the solid surface, the friction coefficient (FC) between solid and liquid was largely reduced with a small amount of methanol and that led to a remarkable decrease of the shear stress. The cause of the FC reduction was investigated in terms of the local rotational diffusion coefficient (RDC) near the solid surface, and it was shown that different from an existing model, the FC and local RDC were not simply inversely proportional to each other in the mixture system because the solid-liquid interfacial state was more complex.
\end{abstract}

DOI: 10.1103/PhysRevE.92.022402

PACS number(s): 68.08.-p, 47.55.-t, 61.20.-p

\section{INTRODUCTION}

The velocity slip between solid and liquid interfaces becomes non-negligible as the characteristic length scale of the flow decreases [1,2]. This velocity slip is commonly quantified as the slip length $l_{\mathrm{s}}$ in the Navier boundary condition,

$$
v_{\mathrm{s}}=l_{\mathrm{s}} \frac{\partial v_{x}}{\partial z},
$$

where $v_{\mathrm{s}}$ and $\partial v_{x} / \partial z$ denote the slip velocity between solid and liquid and gradient of lateral liquid velocity $v_{x}$ along the $z$ direction normal to the surface, respectively. As the slip length is typically at nanometer scale, the slip greatly affects the flow feature in nanoscale channels, and from an engineering point of view, the mass transport can be enhanced by controlling the velocity slip in nanoscale flow systems, e.g., desalination and separation through membranes, liquid transport inside carbon nanotubes, and biological flows [1-3].

Regarding water, the most common liquid in our life, it is reported that the slip length at hydrophobic solid surfaces is in the range of tens of nanometers, while it is largely affected by solid-liquid interfacial conditions such as surface roughness, diffusion coefficient of liquid, and dissolved gas [4]. As an empirical and practical way to change the flow feature of water, various additives are widely used, e.g., wateralcohol mixtures are used in the processes of semiconductor fabrication and printing industry [5,6]. Such liquid mixtures are also common in biological flows. Although there have been several studies on the velocity slip of miscible multicomponent liquids through molecular dynamics analysis using mixtures of monoatomic model fluids $[7,8]$, the effects of mixing of real

\footnotetext{
*nakaoka@gcom.mech.eng.osaka-u.ac.jp

†yamaguchi@mech.eng.osaka-u.ac.jp
}

liquids with multiatomic and polar molecules have not been fully examined yet.

In this paper, we have performed nonequilibrium molecular dynamics simulations of a Couette-type flow of water and methanol mixture liquid between nonpolarized smooth solid surfaces and examined the force balance between shear stress and viscous stress and the friction coefficient for various methanol concentrations. We have also investigated the change of liquid mobility in the vicinity of the solid surface due to mixing in terms of the rotational diffusion coefficient.

\section{SIMULATION METHOD}

\section{A. Potential model}

As the constituent liquid molecules, water $\left(\mathrm{H}_{2} \mathrm{O}\right)$ and methanol $\left(\mathrm{CH}_{3} \mathrm{OH}\right.$, denoted by $\mathrm{MeOH}$ hereafter $)$ molecules are expressed by the SPC/E [9] and the OPLS-UA [10,11] potential models, respectively. Both molecules are treated as a rigid body without intra-atomic degrees of freedom consisting of fixed interaction sites and masses, where $\mathrm{O}, \mathrm{H}$, and $\mathrm{CH}_{3}$ are treated as single interaction sites. In these models, the 12-6 Lennard-Jones (L-J) and Coulomb potentials are applied as the intermolecular potentials between these interaction sites. These interactions are truncated at a cutoff distance of $r_{c}=1.5 \mathrm{~nm}$, and quadric functions are added so that the potentials and interaction forces smoothly become zero at $r_{c}$ [12]. There is no L-J interaction for single hydrogen sites.

An FCC crystal exposing the (111) face is used as the solid wall. The interaction potential between wall atoms is expressed by the harmonic potential for nearest neighbors with a distance of $r_{i j}$ as

$$
\Phi_{\mathrm{H}}\left(r_{i j}\right)=\frac{k}{2}\left(r_{i j}-r_{0}\right)^{2},
$$


TABLE I. Potential and mass parameters for liquid molecules.

\begin{tabular}{lcccc}
\hline \hline & $\sigma(\mathrm{nm})$ & $\varepsilon\left(10^{-21} \mathrm{~J}\right)$ & $q(\mathrm{e})$ & mass $\left(10^{-26} \mathrm{~kg}\right)$ \\
\hline $\mathrm{H}$ (water) & - & - & 0.4238 & 0.1674 \\
$\mathrm{O}($ water $)$ & 0.3166 & 1.08 & -0.8476 & 2.657 \\
$\mathrm{H}(\mathrm{MeOH})$ & - & - & 0.435 & 0.1674 \\
$\mathrm{O}(\mathrm{MeOH})$ & 0.307 & 1.182 & -0.7 & 2.657 \\
$\mathrm{CH}_{3}(\mathrm{MeOH})$ & 0.3775 & 1.439 & 0.265 & 2.497 \\
\hline \hline
\end{tabular}

where $r_{0}$ and $k$ denote the equilibrium distance and spring constant, respectively. The physical properties of platinum are used for the atomic mass and lattice constant of the solid surface [12]. The interactions between solid and liquid atoms are assumed to be only with the L-J potential; i.e., we use a nonpolar solid surface model without Coulomb interaction.

The L-J potential parameters between the molecules of different components are set by the Lorentz-Berthelot mixing rules [13], where the length and energy parameters of the L-J potential $\sigma$ and $\varepsilon$ for particles $i$ and $j$ of different species are, respectively, given by

$$
\sigma_{i j}=\frac{1}{2}\left(\sigma_{i i}+\sigma_{j j}\right)
$$

and

$$
\varepsilon_{i j}=\sqrt{\varepsilon_{i i} \varepsilon_{j j}} .
$$

As described later in Fig. 5, the viscosity dependence of the methanol aqueous solution on the molar concentration qualitatively corresponds to experimental data, which do not linearly change with the molar concentration but take a maximum at a certain value. This correspondence indicates that the basic liquid structure governing the viscosity of the water-methanol mixture is fairly represented by the mixing rules [14].

The L-J parameters of the solid atom $\sigma_{\text {wall-wall }}$ and $\varepsilon_{\text {wall-wall }}$ are used only for the Lorentz-Berthelot mixing rules in the simulation, where the van der Waals radius of a platinum crystal is used for $\sigma_{\text {wall-wall, and }} \varepsilon_{\text {wall-wall }}$ is numerically set so that the contact angle of a single-component water droplet becomes about $90^{\circ}$ [12]. With these parameters, a singlecomponent methanol liquid shows complete wetting on this solid surface, i.e., zero contact angle [12].

The potential and mass parameters of liquid and wall molecules are summarized in Tables I and II, respectively.

\section{B. Simulation system}

Figure 1 exhibits an example of the Couette flow simulation system consisting of water and methanol mixture liquid. The FCC (111) crystal surfaces consisting of three layers are

TABLE II. Potential and mass parameters for wall atom.

\begin{tabular}{lcc}
\hline \hline$\sigma_{\text {wall-wall }}(\mathrm{nm})$ & $\varepsilon_{\text {wall-wall }}\left(10^{-21} \mathrm{~J}\right)$ & \\
\hline $0.35^{\mathrm{a}}$ & $1.44^{\mathrm{a}}$ & \\
$k(\mathrm{~N} / \mathrm{m})$ & $r_{0}(\mathrm{~nm})$ & mass $\left(10^{-26} \mathrm{~kg}\right)$ \\
\hline 46.8 & 0.277 & 32.39 \\
\hline \hline
\end{tabular}

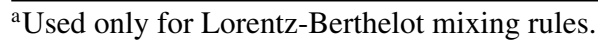

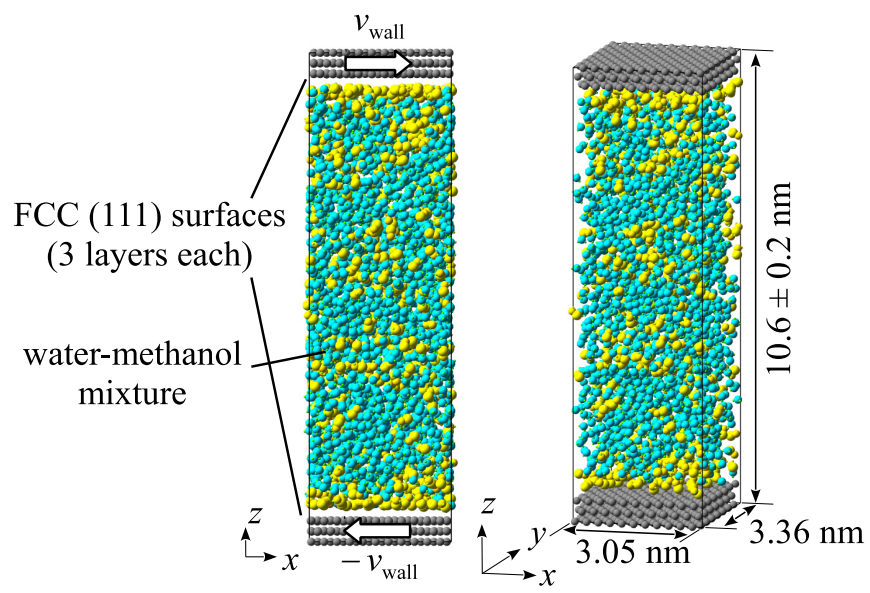

FIG. 1. (Color online) Simulation system of Couette-type flow of water and methanol mixture liquid.

located at the top and bottom of the calculation cell with the periodic boundary conditions in the $x$ and $y$ directions. The size of the calculation cell in the $x$ and $y$ directions is about $3.05 \times 3.36 \mathrm{~nm}^{2}$. The system height is fixed in the range of 10.6 $\pm 0.2 \mathrm{~nm}$ depending on the methanol concentration, where the height is determined through the preliminary simulations in which the pressure is controlled to approximately attain the atmospheric pressure by imposing an external force on the top wall [15].

Single-component water or methanol or a mixture of water and methanol is confined between two walls consisting of 462 atoms for each. The numbers of liquid molecules for water and methanol single-component systems are 3160 and 1336, respectively, while the numbers for mixtures with different water-methanol ratios are chosen to give approximately the same volume under the atmospheric pressure. The relative positions of the wall atoms in each outmost layer are fixed, and the temperature is controlled at $300 \mathrm{~K}$ by applying the Langevin method to the wall atoms in each middle layer so that the heat generated by the solid-liquid friction and viscous dissipation in the liquid is removed through the walls [16]. We have checked that the $y$ and $z$ directional local temperatures of liquid calculated in parallel bins along the $z$ direction are kept constant at the control temperature throughout the system.

The flow is driven by moving all the atoms of top and bottom walls, respectively, to the $x$ and $-x$ directions at a constant speed of $v_{\text {wall }}=20 \mathrm{~m} / \mathrm{s}$. We have preliminary examined the influence of the wall speed on the friction coefficient described later in the range between 2.5 and $40 \mathrm{~m} / \mathrm{s}$ for several methanol fractions and confirmed that the friction coefficient remains at the same order although the fluctuation increases as the wall speed decreases [4].

The velocity Verlet method with the modified quaternion constraint techniques $[17,18]$ is applied for the integration of the equation of motion with a time step of $1 \mathrm{fs}$. A steady Couette flow is obtained through the relaxation run of over $5 \mathrm{~ns}$, and the time average of $40 \mathrm{~ns}$ is used for the data analysis. The error bars in the following figures are plotted as the standard error of $1 \mathrm{~ns}$ average data segments of the entire simulation data, except the viscosity in Fig. 5 (see Appendix). 


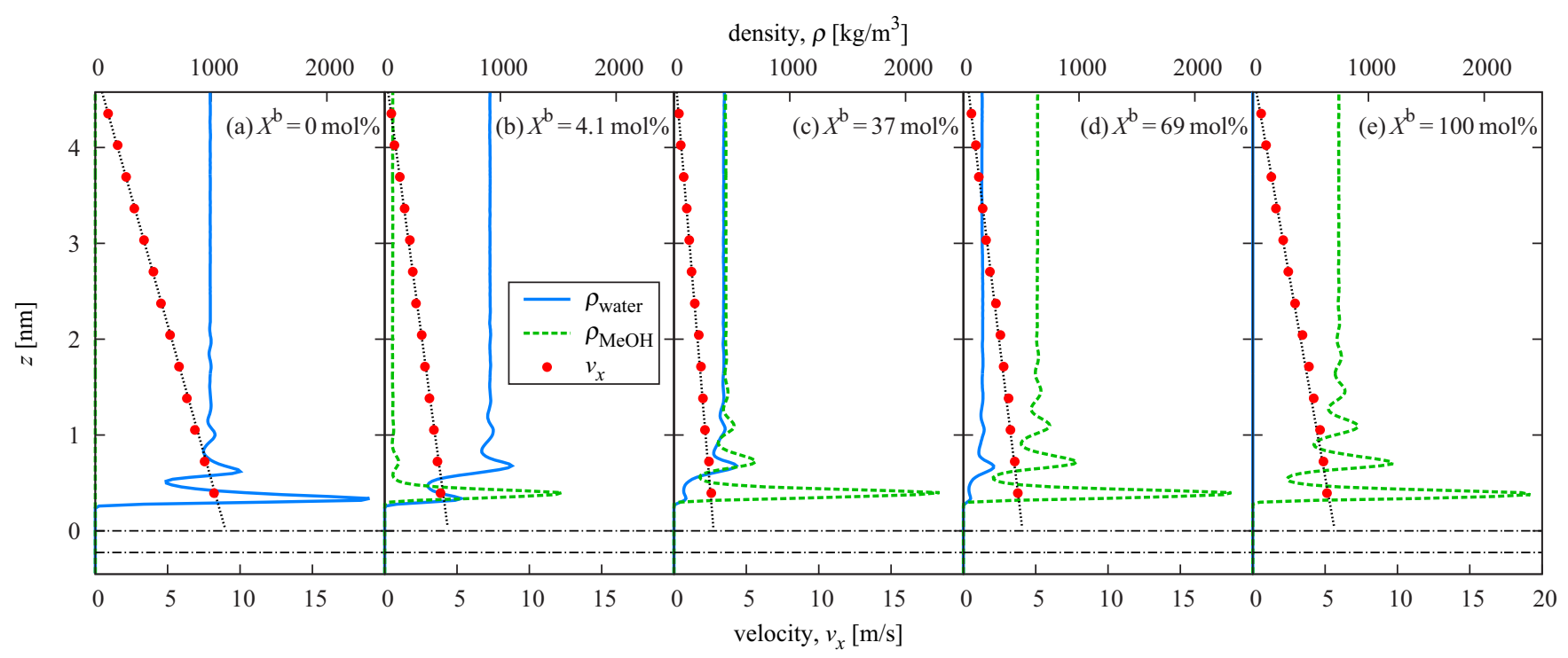

FIG. 2. (Color online) Distributions of water and methanol densities $\rho_{\text {water }}$ (blue solid line) and $\rho_{\text {MeOH }}$ (green dashed line), respectively, and lateral velocity along the $x$ direction $v_{x}$ (red dots) for different methanol molar fractions in the bulk region $X^{\mathrm{b}}$ of (a) $0 \%$, (b) $4.1 \%$, (c) $37 \%$, (d) $69 \%$, and (e) $100 \%$. The dash-dot black lines correspond to the mean position of solid layers, and the origin of the $z$ axis is set as the mean position of the innermost layer of the solid surface. The dotted black line along the red dots is a fitting line of the velocity in the bulk.

\section{RESULTS AND DISCUSSION}

\section{A. Density and velocity distributions}

The distributions of the water and methanol densities $\rho_{\text {water }}$ and $\rho_{\mathrm{MeOH}}$, respectively, and lateral velocity along the $x$ direction $v_{x}$ with different compositions are shown in Fig. 2 as a function of the distance from the solid surface, where the origin of the $z$ axis is set at the mean position of each innermost solid layer. The distributions are calculated using the center of mass position and velocity of water and methanol molecules and merged for the top and bottom by taking the system symmetry into account.

The water and methanol densities are constant for about $z>2.5 \mathrm{~nm}$, and this means that water and methanol molecules are uniformly mixed to form a bulk region away from the solid surface. The methanol molar fraction in the bulk is denoted by $X^{\mathrm{b}}$ as a parameter for the following discussion. Meanwhile, in the vicinity of the solid surface, adsorption layers with a high density are observed in all compositions. It is remarkable that, even with a small methanol fraction in the bulk, the methanol molecules form the first adsorption layer near the solid surface.

Regarding the lateral velocity distribution, it is approximately linear in the bulk region as seen with the dotted black lines fitted for this region. This feature is basically the same as in a macroscopic Couette flow while the distribution slightly deviates from the linear one inside the adsorption layers. In addition, the lateral liquid velocity at the bottom is smaller than the wall velocity of $20 \mathrm{~m} / \mathrm{s}$ in all cases, showing that a distinct velocity slip exists at the solid-liquid interface.

Figure 3 shows the methanol molar fraction in the first adsorption layer $X^{\mathrm{a}}$ and slip length $l_{\mathrm{s}}$ plotted against the methanol molar fraction in the bulk $X^{\mathrm{b}}$, where the first adsorption layer is defined as the region between the solid surface and the height at which the density of the liquid mixture takes the first local minimum. On the other hand, the slip length is calculated by Eq. (1) using the slip velocity $v_{\mathrm{s}}$ and velocity gradient $\partial v_{x} / \partial z$ in Fig. 2. Specifically, the latter is obtained as the gradient of the linear fitting line to the bulk velocity in Fig. 2, and the former is the difference between the wall velocity and the velocity value on the fitting line at the solid surface of $z=0$. The velocity in the adsorption layers is not used to determine the slip length because we intend to treat the Navier boundary condition for macroscopic hydrodynamics in Eq. (1) which includes all the effects of microscopic adsorption layers [19].

As mentioned above, $X^{\mathrm{a}}$ is always higher than $X^{\mathrm{b}}$ in the mixture systems, and this adsorption of methanol onto the solid surface can be explained by the property that the

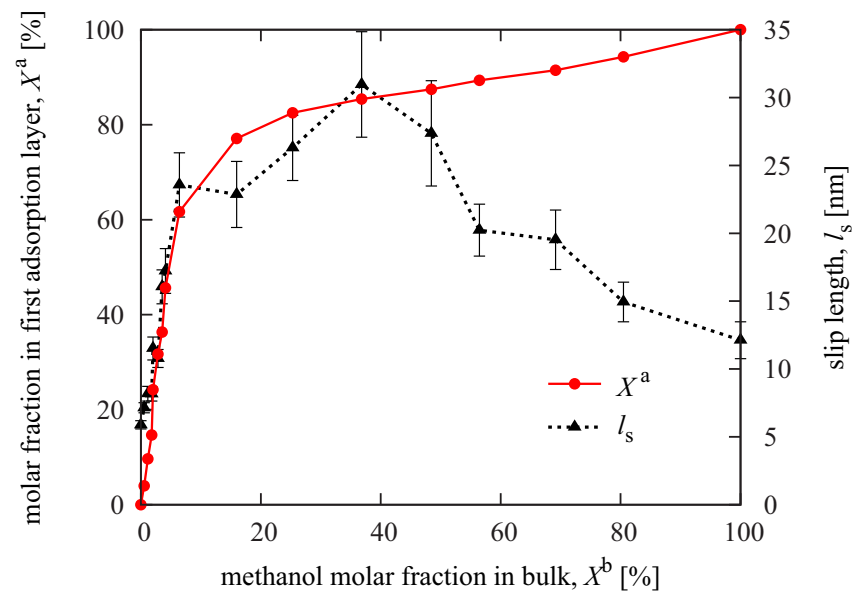

FIG. 3. (Color online) Methanol molar fraction in the first adsorption layer $X^{\mathrm{a}}$ and slip length $l_{\mathrm{s}}$ for various methanol molar fractions in the bulk $X^{\mathrm{b}}$. The slip length is calculated by Eq. (1) using the slip velocity and velocity gradient obtained in Fig. 2. 
hydrophobic methyl group $\mathrm{CH}_{3}$ of methanol tends to gather onto the nonpolar surface rather than to diffuse into the bulk. It is also observed that the increase of $X^{\mathrm{a}}$ is steep for a lower bulk fraction $X^{\mathrm{b}}$ below $10 \%$, and it is relatively moderate thereafter. Regarding the slip length $l_{\mathrm{s}}$, it steeply rises up as the $X^{\mathrm{b}}$ increases up to about $X^{\mathrm{b}}=10 \%$, and this steep increase is similar to that of $X^{\mathrm{a}}$. It should be mentioned that a relatively large slip length is achieved by a small amount of methanol molecules because they are adsorbed on the solid surface and change the solid-liquid interfacial condition in the present case of a nonpolarized solid surface. For a higher $X^{\mathrm{b}}$ value, the slip length $l_{\mathrm{s}}$ decreases with the increase of $X^{\mathrm{b}}$ in contrast to the gradual increase of $X^{\mathrm{a}}$ there. This contrast indicates the fact that the slip length dose not purely reflect the interfacial property but is also affected by the viscosity of liquid bulk [19-21].

\section{B. Force balance between shear stress and viscous stress}

As shown in the previous section, methanol mixing changes both properties of the liquid bulk and solid-liquid interface, and both simultaneously affect the slip. In order to independently evaluate these contributions, we have carried out further analysis on the bulk viscosity through the lateral force balance between the shear stress and viscous stress in the bulk. In addition we have examined the validity of the viscosity calculated based on the Green-Kubo relation.

The shear stress $\tau$ in the system is plotted against $X^{\mathrm{b}}$ in Fig. 4, where the $\tau$ values are independently calculated using the following three methods: (1) $\tau_{\text {wall }}$ as the force exerted on the wall divided by the surface area, (2) $\tau_{\text {bulk }}$ as the average shear stress inside the bulk region, and (3) $\tau_{\text {vis }}$ as the viscous stress in the bulk region evaluated based on the Newton's law of viscosity. In more detail, for the second method, the bulk shear stress $\tau_{\text {bulk }}$ is obtained by averaging the $x z$ and $z x$ components of the local stress tensor $\sigma$ in the bulk region. The stress tensor

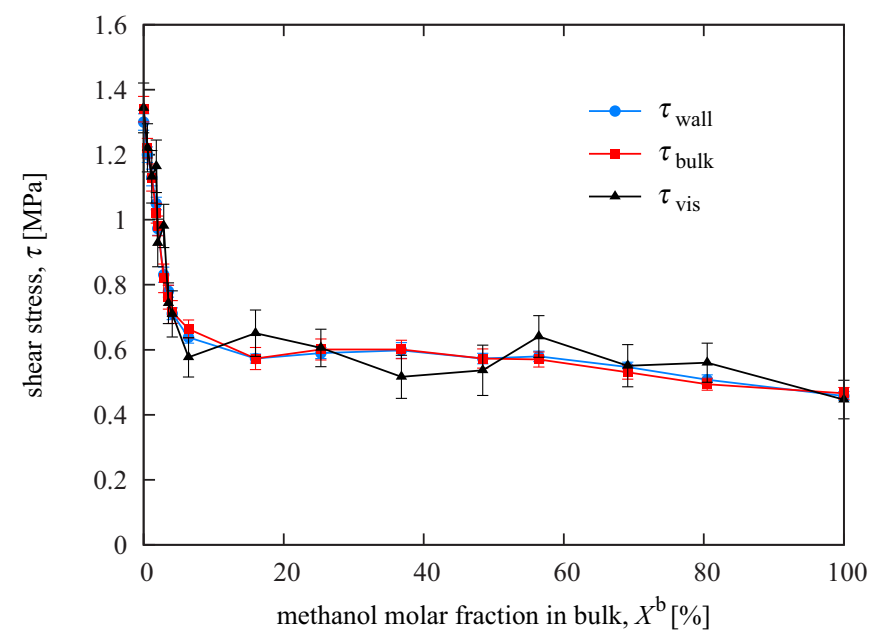

FIG. 4. (Color online) Relations between methanol molar fraction in the bulk $X^{\mathrm{b}}$ and lateral shear stress $\tau$. The three $\tau$ values $\tau_{\text {wall }}$, $\tau_{\text {bulk }}$, and $\tau_{\text {vis }}$ are independently calculated using the force exerted on the wall, the average shear stress inside the bulk region, and the viscous stress in the bulk region $\tau_{\text {vis }}$ evaluated using the present velocity gradient in the bulk region and viscosity, respectively. $\sigma$ inside a local region with a volume $V$ is calculated based on the weighted virial theorem $[22,23]$,

$$
\boldsymbol{\sigma}=-\frac{1}{V}\left\langle\sum_{i \in V} m_{i} \mathbf{v}_{i} \otimes \mathbf{v}_{i}\right\rangle-\frac{1}{V}\left\langle\sum_{i}^{N} \sum_{j(>i)}^{N} w_{i j} \mathbf{r}_{i j} \otimes \mathbf{f}_{i j}\right\rangle,
$$

where $m_{i}$ and $\mathbf{v}_{i}$ respectively are the mass and velocity of molecule $i$, and $\mathbf{r}_{i j}$ and $\mathbf{f}_{i j}$, respectively, denote the relative position vector of the center of mass and the intermolecular force vector between molecules $i$ and $j$. The weighting function $w_{i j}$ is the length fraction of the line segment $\left|\mathbf{r}_{i j}\right|$ contained in the local region. For the third method, the viscous stress in the bulk is calculated by the product of the viscosity $\mu$ and velocity gradient in the bulk region as

$$
\tau_{\mathrm{vis}}=\mu \frac{\partial v_{x}}{\partial z},
$$

where $\mu$ denotes the viscosity and $\partial v_{x} / \partial z$ is the velocity gradient in the bulk region of the present Couette flows. The latter is obtained as the gradient of the dotted black fitting lines in Fig. 2, while the former is calculated from the fluctuation of the off-diagonal components of the pressure tensor based on the Green-Kubo relation in each independent equilibrium homogeneous bulk system whose methanol molar fraction is set equal to the corresponding $X^{\mathrm{b}}$ value in the Couette flow system. The calculation details of the viscosity are shown in Appendix, and its dependence on the molar fraction is described later.

As shown in Fig. 4, the three independently evaluated shear stresses basically result in the same value within the range of error bars. The correspondence between the first and second shear stress values means that the two forces exerted on the adsorption layer, i.e., one from the wall $\tau_{\text {wall }}$ and the other from the bulk $\tau_{\text {bulk }}$, are balanced in this steady Couette flow system. In addition, the correspondence between the second and third shear stresses, i.e., between $\tau_{\text {bulk }}$ and $\tau_{\text {vis }}$, indicates that the Newton's law of viscosity holds in this system even at a high shear rate of $\sim 10^{9} \mathrm{~s}^{-1}$, in other words, the water-methanol mixture behaves as a Newtonian liquid. In terms of the shear rate, the Newton's law of viscosity is only applicable at a lower shear rate range according to the Green-Kubo relation, and the range is roughly estimated by the inverse of the relaxation time [25]. This relaxation time is evaluated from the autocorrelation function of the pressure tensor. As shown in the Appendix, the relaxation time in the equilibrium water-methanol mixture systems is at the order of $10^{-14}-10^{-13} \mathrm{~s}$, and from this estimate, the present shear rate of $10^{9} \mathrm{~s}^{-1}$ is considered to be still in the Newtonian range.

The two components of Newtonian viscous stress in Eq. (6) is reevaluated here in order to clarify the effect of mixing on the bulk viscosity $\mu$, and also to check the validity of the potential model adopted in this study. Figure 5 shows the viscosity $\mu$ and velocity gradient $\partial v_{x} / \partial z$ plotted against the methanol molar fraction in the bulk $X^{\mathrm{b}}$. As mentioned above, the viscosity is calculated in independent equilibrium homogeneous bulk systems described in Appendix, and the experimental viscosity value at $303.15 \mathrm{~K}$ [24] is also shown for comparison. The calculated viscosity gradually increases upon the increase of the methanol molar fraction in the range around $0 \leqslant X^{\mathrm{b}} \leqslant$ $30 \%$ and decreases for $X^{\mathrm{b}} \geqslant 30 \%$. Although the calculated 


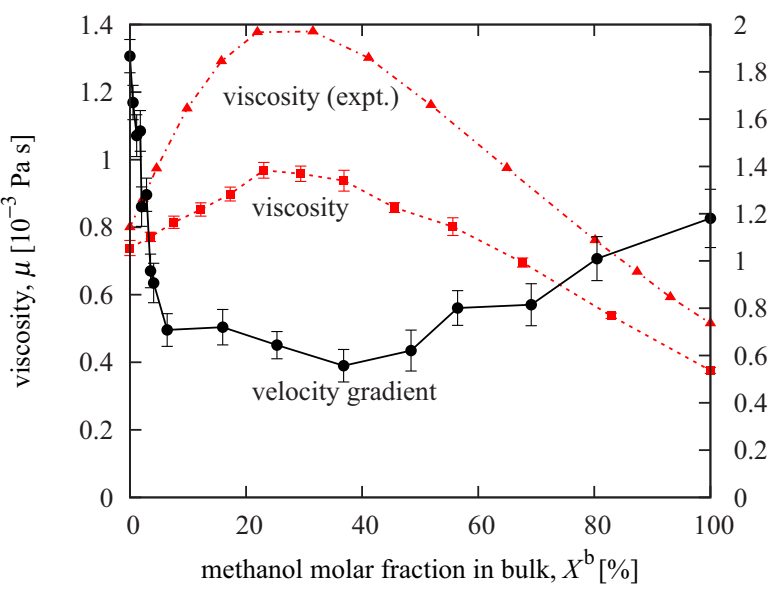

FIG. 5. (Color online) Viscosity of water and methanol mixture and velocity gradient in the bulk region of Couette flow as a function of methanol molar fraction in the bulk $X^{\mathrm{b}}$. The viscosity is calculated in independent equilibrium homogeneous bulk systems with a corresponding methanol molar fraction. Experimental data of viscosity at $303.15 \mathrm{~K}$ are also plotted [24].

viscosity is lower than the experimental one especially in the mixture, the characteristic curve taking the maximum at a molar fraction $X^{\mathrm{b}}$ around $30 \%$ is reproduced in the simulation, and this implies that the characteristic feature of water-methanol mixture is simulated by the present molecular models.

In contrast to the gradual change of the viscosity, the velocity gradient largely decreases as $X^{\mathrm{b}}$ increases up to about $10 \%$. These results indicate that the steep decrease of the shear stress for about $X^{\mathrm{b}}<10 \%$ observed in Fig. 4 is not due to the change in the viscosity but due to the decrease of the velocity gradient, which results from the enhancement of the velocity slip through the adsorption of methanol molecules on the solid surface.

\section{Friction coefficient}

The slip length $l_{\mathrm{s}}$ in the Navier boundary condition Eq. (1) is affected by the viscosity $\mu$ and therefore does not purely reflect the interfacial property [19-21]. By eliminating the velocity gradient $\partial v_{x} / \partial z$ from Eqs. (1) and (6), the Navier boundary condition is rewritten

$$
\tau=\lambda v_{\mathrm{s}},
$$

as the relation between the shear stress and slip velocity. The coefficient $\lambda$ is related to the slip length $l_{\mathrm{s}}$ as

$$
l_{\mathrm{s}}=\frac{\mu}{\lambda}
$$

and is called the friction coefficient (FC). As simply shown in Eq. (8), the FC characterizes the interfacial condition, e.g., a larger FC basically means a strong interaction between solid and liquid with a shorter slip length, and is usually considered to be independent of the bulk property [19-21].

Figure 6 shows the friction coefficient $\lambda$ for different methanol fractions in the bulk $X^{\mathrm{b}}$, where the FC is calculated by Eq. (7) using the $v_{\mathrm{s}}$ and $\tau_{\text {wall }}$ values. Two slip length values obtained from Eqs. (8) and (1), of which the latter is identical

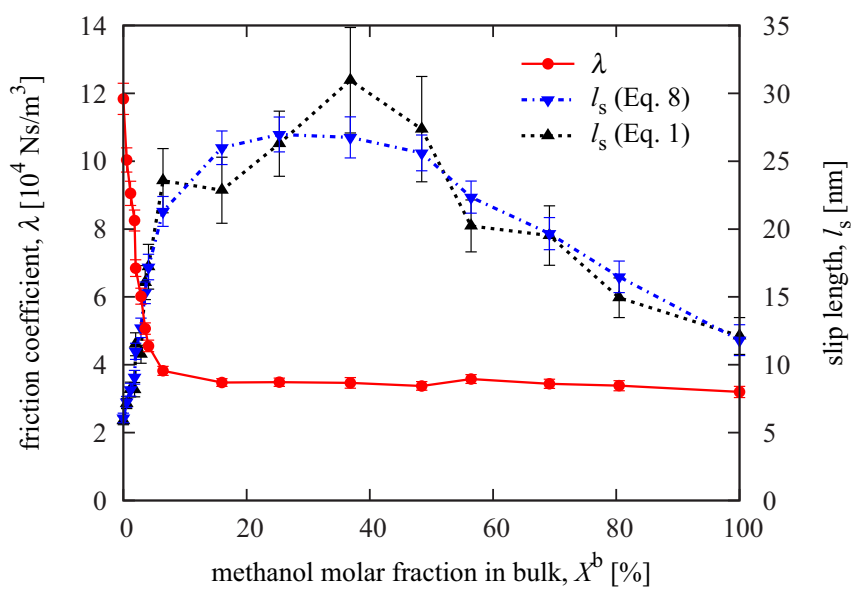

FIG. 6. (Color online) Friction coefficient (FC) in the Navier boundary condition in Eq. (7) and slip length given by Eq. (8) for various methanol molar fractions in the bulk. The slip length given by Eq. (1) shown in Fig. 3 is also plotted.

to that shown in Fig. 3, are also plotted. The FC decreases by about one third when $X^{\mathrm{b}}$ increases from 0 to $10 \%$. This change in the FC is the main cause of the steep decrease of the shear stress in Fig. 4 induced by a slight increase in the methanol fraction. Regarding the slip length, two differently calculated $l_{\mathrm{s}}$ values are consistent with each other though the latter obtained from Eq. (1) fluctuates more than the former obtained from Eq. (8) because the velocity gradient shown in Fig. 5 includes relatively large error.

Here the relation between the FC and solid-liquid interfacial energy $\gamma_{\mathrm{sl}}$ is discussed more in detail. Huang et al. [26] evaluated the slip length of water on various solid surfaces with different wettability using MD simulations and showed that the slip length increased as the droplet contact angle of the corresponding system increased. Considering that the liquid-vapor interfacial energy $\gamma_{1 \mathrm{v}}$ in Young's equation [27]

$$
\gamma_{\mathrm{sl}}-\gamma_{\mathrm{sv}}=-\gamma_{\mathrm{lv}} \cos \theta
$$

is unchanged for their cases using only water as the liquid, and also considering that the viscosity $\mu$ in Eq. (8) is unchanged there as well, their result implies that the FC increases when the solid-liquid affinity becomes higher with the decrease of the solid-liquid interfacial energy relative to the solid-vapor interfacial energy $\gamma_{\mathrm{sl}}-\gamma_{\mathrm{sv}}$. However, our results indicate that it is not the case for the present water-methanol mixture system on a nonpolarized surface; indeed the FC decreases with the increase of the methanol fraction as shown in Fig. 6, but the $\gamma_{\mathrm{sl}}$ of a water-methanol mixture decreases as shown in the left graph of Fig. 9 of our previous study [12]. In addition, while the $\mathrm{FC}$ in the present study is almost constant when the molar fraction $X^{\mathrm{b}}$ is larger than about $10 \%$, it has been shown that $\gamma_{\mathrm{s} 1}$ gradually decreases at this range in Fig. 9 of Ref. [12], corresponding to the mass fraction range $f_{\mathrm{MeOH}}^{\text {bulk }}>$ $16 \%$ there. This discrepancy implies that the correlation between the FC and solid-liquid interfacial energy for a given single-component liquid on various solid surfaces [26] cannot simply be applied for mixture liquids of two given molecules with different molar fractions on a given solid surface. In fact, the negative correlation between the FC and $\gamma_{\mathrm{sl}}$ does not hold 
even for the comparison between pure, i.e., single-component water and methanol on the same nonpolarized solid surface used in the present study. It has been shown in the left graph of Fig. 9 of Ref. [12] that the solid-liquid interfacial energy $\gamma_{\mathrm{sl}}$ of water at $f_{\mathrm{MeOH}}^{\text {bulk }}=0$ in this graph is higher than that of single component methanol depicted by the red line there; however, the friction coefficient $\lambda$ of pure water at $X^{b}=0 \%$ in Fig. 6 in the present study is higher than that of pure methanol at $X^{\mathrm{b}}=$ $100 \%$.

Finally, we discuss here about the fast decrease and plateauing of the friction coefficient $\lambda$ with the increase of the methanol fraction in bulk $X^{\mathrm{b}}$. Considering that the methanol molecules tend to be adsorbed onto the solid surface as shown in Fig. 3 , this change in $\lambda$ could be explained by the fact that methanol molecules form the first adsorption layer even with a small methanol fraction in the bulk, so that locally the solid-liquid interface is very similar to the one of pure methanol. However, we have carefully observed the methanol orientation in the adsorption layer and found that the methanol orientation is strongly biased there due to the formation of a hydrogen-bond network with the water molecules in the bulk region and is largely different from the solid-liquid interface structure of pure methanol at $X^{\mathrm{b}}=100 \%$. Further analysis on the relation between this microscopic molecular structure and the FC is a future target of this study.

\section{Rotational diffusion coefficient}

Up to this point, it has been shown that the FC is largely reduced by adding a small amount of methanol molecules which are adsorbed on the solid surface. In order to examine the microscopic mechanism of this FC reduction, the local motion of the molecules at the solid-liquid interface is investigated more in detail in terms of the molecular rotational motion here.

According to Bocquet and Barrat $[28,29]$ and Sendner et al. [4], the FC is estimated from an approximation based on the Green-Kubo relation

$$
\lambda=\frac{1}{A k_{\mathrm{B}} T} \int_{0}^{\infty}\left\langle F_{\mathrm{w}}(t) F_{\mathrm{w}}(0)\right\rangle_{\mathrm{eq}} d t \approx \frac{\tau_{\mathrm{r}}\left\langle F_{\mathrm{w}}^{2}\right\rangle_{\mathrm{eq}}}{A k_{\mathrm{B}} T},
$$

where $A, F_{\mathrm{w}}$, and $\tau_{\mathrm{r}}$ are the surface area, total lateral force exerted on the wall by the liquid, and relaxation time, respectively, and \langle\rangle$_{\text {eq }}$ denotes the equilibrium ensemble average. The relaxation time $\tau_{\mathrm{r}}$ is generally given by

$$
\tau_{\mathrm{r}} \sim \frac{\sigma^{2}}{D_{\mathrm{t}}}
$$

where $\sigma$ is the characteristic length scale and $D_{\mathrm{t}}$ is the translational diffusion coefficient (TDC) of liquid near the solid surface [4]. Thus, from Eqs. (10) and (11), the FC is roughly estimated to be

$$
\lambda \sim \frac{\sigma^{2}\left\langle F_{\mathrm{w}}^{2}\right\rangle_{\mathrm{eq}}}{A k_{\mathrm{B}} T D_{\mathrm{t}}} .
$$

Equation (12) implies that the FC is inversely proportional to the TDC of the molecules at the solid surface; i.e., the liquid easily slips when the liquid molecules near the surface move easily.
In order to investigate the local liquid mobility near the solid surface, we have calculated the rotational diffusion coefficient (RDC) of liquid molecules in the first adsorption layer instead of the TDC. In our previous study on pure water, we have shown that the RDC of water basically has the same information as the TDC in homogeneous systems as well as in heterogeneous systems with solid surface, and we also have shown that for calculating the spatial distribution with a higher resolution, the RDC is advantageous over the TDC because the relaxation time of the rotational motion of water molecules evaluated by the autocorrelation function of the angular velocity is much shorter than that of the translational motion [30]. This feature is owing to the fact that water molecules have a small inertia moment. This can also be applied to methanol molecules with a small inertia moment, and we make use of this property to the present study.

According to the Einstein's diffusion equation, the mean square of angular displacement $s$ under Brownian motion is proportional to the elapsed time $t$ as

$$
\left\langle s^{2}\right\rangle=4 D_{\mathrm{r}} t
$$

where $D_{\mathrm{r}}$ is the RDC [30,31].

In order to relate $D_{\mathrm{r}}$ to $D_{\mathrm{t}}$, we assumed that both the StokesEinstein relation

$$
D_{\mathrm{t}}=\frac{k_{\mathrm{B}} T}{6 \pi \mu \sigma}
$$

and Stokes-Einstein-Debye relation [30,32]

$$
D_{\mathrm{r}}=\frac{k_{\mathrm{B}} T}{8 \pi \mu \sigma^{3}}
$$

hold around the solid surface and that they have the same characteristic length scale $\sigma$. We have shown that the latter relation of Eq. (15) holds at a nonpolarized surface for water [30]. Using Eqs. (14) and (15), Eq. (12) can be rewritten in terms of the local RDC as

$$
\lambda \sim \frac{3\left\langle F_{\mathrm{w}}^{2}\right\rangle_{\mathrm{eq}}}{4 A k_{\mathrm{B}} T D_{\mathrm{r}}} .
$$

This means that the FC is inversely proportional to the local RDC as well if the Green-Kubo relation for the FC in Eq. (10) is applicable.

In order to examine the relation between the FC and local RDC, the RDCs of the water and methanol molecules in the first adsorption layer as well as those in the bulk region are shown in Fig. 7, where the RDCs are plotted as a function of the methanol molar fraction (a) in the bulk $X^{\mathrm{b}}$ and (b) in the first adsorption layer $X$ a . The superscripts "a" and "b" on $D_{\mathrm{r}}$ denote the values in the first adsorption layer and bulk, respectively, and only $D_{\mathrm{r}}^{\mathrm{a}}$ is shown with the friction coefficient $\lambda$ in Fig. 7(b). The average RDCs of water and methanol molecules indicated by the superscripts "ave" in the bulk and in the first adsorption layer are also plotted in Fig. 7 as a rough measure, which are respectively defined by

$$
D_{\mathrm{r}}^{\mathrm{b}, \text { ave }}=\left(1-X^{\mathrm{b}}\right) D_{\mathrm{r}}^{\mathrm{b}, \text { water }}+X^{\mathrm{b}} D_{\mathrm{r}}^{\mathrm{b}, \mathrm{MeOH}},
$$

and

$$
D_{\mathrm{r}}^{\mathrm{a}, \mathrm{ave}}=\left(1-X^{\mathrm{a}}\right) D_{\mathrm{r}}^{\mathrm{a}, \text { water }}+X^{\mathrm{a}} D_{\mathrm{r}}^{\mathrm{a}, \mathrm{MeOH}} .
$$



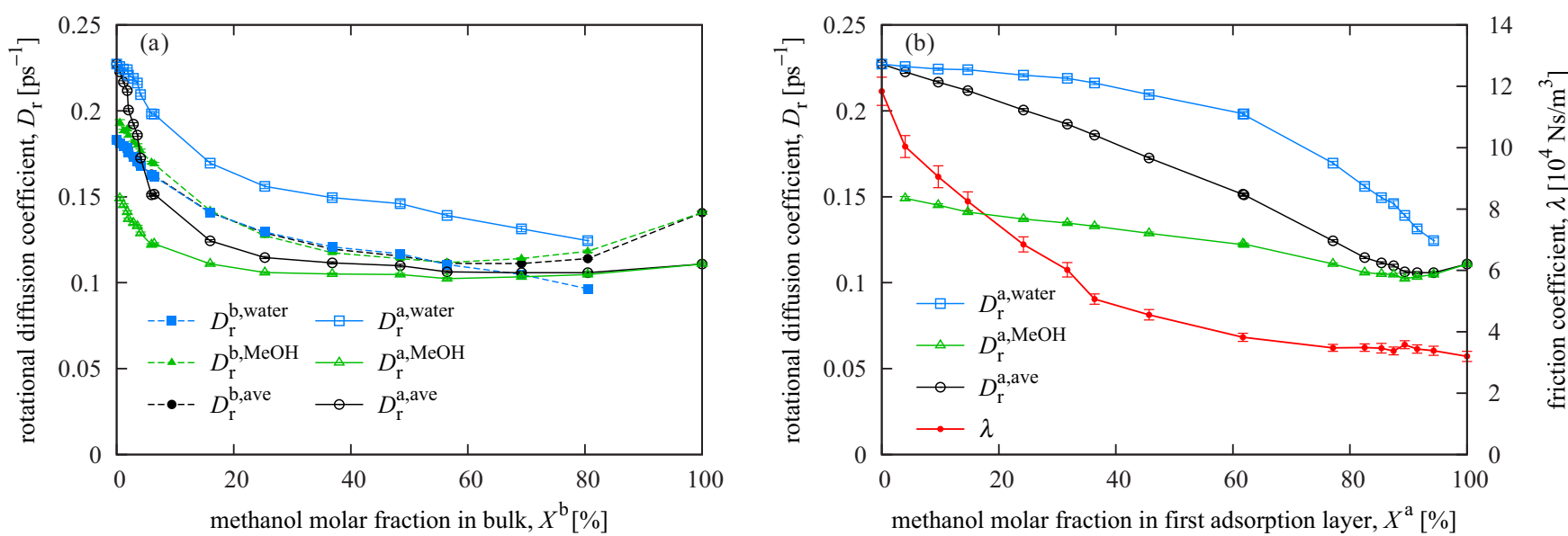

FIG. 7. (Color online) Rotational diffusion coefficient (RDC) $D_{\mathrm{r}}$ as a function of methanol molar fraction $X$ in the bulk and in the first adsorption layer. Superscripts "b" and "a," respectively, denote the local regions of bulk and the first adsorption layer, and "water," and "MeOH," and "ave" for $D_{\mathrm{r}}$ mean the corresponding molecules and their average. Each RDC is plotted against (a) $X^{\mathrm{b}}$ and (b) $X^{\mathrm{a}}$, where the friction coefficient $\lambda$ is also displayed in (b).

Over the whole bulk methanol molar fraction $X^{\mathrm{b}}$ in Fig. 7(a), the RDC of water molecules $D_{\mathrm{r}}^{\text {a,water }}$ in the adsorption layer is higher than that in the bulk $D_{\mathrm{r}}^{\mathrm{b} \text {,water }}$, while the RDC of methanol molecules is on the contrary, i.e., $D_{\mathrm{r}}^{\mathrm{a}, \mathrm{MeOH}}$ is lower than $D_{\mathrm{r}}^{\mathrm{b}, \mathrm{MeOH}}$. In other words, the rotational motion of water molecules is enhanced near the solid surface while that of methanol molecules is reduced there. Another point to be noted is that the $D_{\mathrm{r}}^{\mathrm{b}}-X^{\mathrm{b}}$ relation in Fig. $7(\mathrm{a})$ and $D_{\mathrm{r}}^{\mathrm{a}}-X^{\mathrm{a}}$ relation in Fig. 7(b) are clearly different. These two differences, i.e., between the $D_{\mathrm{r}}^{\text {water }}$ enhancement and $D_{\mathrm{r}}^{\mathrm{MeOH}}$ reduction near the solid surface, and between $D_{\mathrm{r}}^{\mathrm{b}}-X^{\mathrm{b}}$ and $D_{\mathrm{r}}^{\mathrm{a}}-X^{\mathrm{a}}$ relations in the bulk and near the solid surface, imply that the RDC in the adsorption layer is highly affected by the mixing components as well as by the surface properties and cannot be estimated simply from the RDC dependence on the molar fraction in the bulk.

Regarding the relation between $D_{\mathrm{r}}$ and $\lambda$ in Fig. 7(b), they are not inversely correlated as mentioned above in Eq. (16). We have to further investigate carefully the reason of the discrepancy including the applicability of the G-K relation in Eq. (10) to our mixture system, however; it is still unclear. A possible reason is that the total lateral force $\left\langle F_{\mathrm{w}}^{2}\right\rangle_{\mathrm{eq}}$ in Eq. (16) is largely affected by the interfacial state including the density, molecular size and orientation, and resulting solid-liquid interfacial energy, which is more complex in water-methanol mixture systems [12], and this may hinder the effect of the molecular mobility on the FC. This complexity is also indicated in the change of the local RDC near the solid surface mentioned above with Fig. 7, and in order to further model the effects of mixing on the FC, microscopic structures of liquid molecules around the interfaces need to be considered $[1,4,20,21]$.

\section{CONCLUSION}

In order to investigate the effect of mixing on velocity slip, nonequilibrium steady molecular dynamics simulations of a nanoscale Couette flow of water-methanol liquid mixture were performed. Methanol molecules showed a tendency to be adsorbed on the solid surface rather than to diffuse into the bulk liquid, although methanol and water molecules were uniformly mixed in the bulk. Similar to a macroscale Couette flow, the shear stress exerted on the solid surface was equal to the shear stress in the liquid, showing that the mechanical balance holds in nanoscale. In addition, the shear stress in the liquid bulk was equal to the viscous stress, which is a product of the viscosity and velocity gradient. When more methanol molecules were adsorbed on the solid surface, the FC between solid and liquid was largely decreased, and that led to a remarkable decrease of the shear stress. The cause of this FC reduction was investigated in terms of the local RDC near the solid surface, and it was shown that differently from an existing model, the FC and local RDC was not inversely proportional to each other in the mixture system because the solid-liquid interfacial state was more complex.

\section{ACKNOWLEDGMENTS}

S.N. is supported by JSPS KAKENHI Grant No. 14J00811, and Y.Y. is supported by JSPS KAKENHI Grant No. 25420123. We also thankful for fruitful discussion with Donatas Surblys in our group.

\section{APPENDIX: VISCOSITY OF WATER AND METHANOL MIXTURE}

The shear viscosity of water and methanol mixture shown in Fig. 5 is obtained in an equilibrium bulk mixture system in which water and methanol molecules are confined in a cubic calculation cell with the periodic boundary conditions imposed in all directions. The viscosity $\mu$ is calculated from the off-diagonal components of the pressure tensor $P_{\xi \zeta}$ using the Einstein form $[33,34]$

$$
\mu=\frac{V}{2 k_{\mathrm{B}} T} \lim _{t \rightarrow \infty} \frac{1}{t}\left\langle\left(\int_{0}^{t} P_{\xi \zeta}(\tau) d \tau\right)^{2}\right\rangle_{\mathrm{eq}}[\xi, \zeta(\neq \xi)=x, y, z],
$$


where $V, k_{\mathrm{B}}$, and $T$ denote the volume, Boltzmann constant and temperature, respectively. The pressure tensor $\mathbf{P}$ is calculated by the virial theorem,

$$
\mathbf{P}=\frac{1}{V}\left(\sum_{i} m_{i} \mathbf{v}_{i} \otimes \mathbf{v}_{i}+\sum_{i, j(>i)} \mathbf{r}_{i j} \otimes \mathbf{f}_{i j}\right),
$$

where $m_{i}$ and $\mathbf{v}_{i}$ denote the mass and velocity of molecule $i$, respectively, and $\mathbf{r}_{i j}$ and $\mathbf{f}_{i j}$ are the relative position and force vectors from $i$ to $j$, respectively. Temporal average of $36 \mathrm{~ns}$ is performed in a system under constant $N V E$ condition, which is achieved after a preliminary equilibration under constant NPT condition [35] at a control temperature of $300 \mathrm{~K}$ with the atmospheric pressure. The system contains 1200 molecules for a single-component water system and 500 molecules for a single-component methanol system, while the numbers for mixtures with different water-methanol ratios are chosen to give approximately the same volume as the single-component systems. The error bars in Fig. 5 are plotted as the standard error of the mean values from 3 ns segments of the entire simulation data.

The relaxation time is evaluated by rewriting Green-Kubo form of the viscosity as

$$
\mu=\frac{V}{k_{\mathrm{B}} T} \int_{0}^{\infty}\left\langle P_{\xi \zeta}(0) P_{\xi \zeta}(t)\right\rangle_{\mathrm{eq}} d t \equiv \frac{V \tau_{\mathrm{v}}\left\langle P_{\xi \zeta}^{2}\right\rangle_{\mathrm{eq}}}{k_{\mathrm{B}} T}
$$

where $\tau_{\mathrm{v}}$ is the relaxation time defined by

$$
\tau_{\mathrm{v}}=\frac{\int_{0}^{\infty}\left\langle P_{\xi \zeta}(0) P_{\xi \zeta}(t)\right\rangle_{\mathrm{eq}} d t}{\left\langle P_{\xi \zeta}^{2}\right\rangle_{\mathrm{eq}}} .
$$

The calculated $\tau_{\mathrm{v}}$ value takes the maximum for a mixture system at a methanol molar fraction around $50 \mathrm{~mol} \%$ of about $105 \mathrm{fs}$ and those for single water and methanol systems are 58 and $71 \mathrm{fs}$, respectively.
[1] L. Bocquet and E. Charlaix, Chem. Soc. Rev. 39, 1073 (2010).

[2] L. Bocquet and P. Tabeling, Lab Chip 14, 3143 (2014).

[3] H. G. Park and Y. Jung, Chem. Soc. Rev. 43, 565 (2014).

[4] C. Sendner, D. Horinek, L. Bocquet, and R. R. Netz, Langmuir 25, 10768 (2009).

[5] J.-G. Park, S.-H. Lee, J.-S. Ryu, Y.-K. Hong, T.-G. Kim, and A. A. Busnaina, J. Electrochem. Soc. 153, G811 (2006).

[6] D. Peter, M. Dalmer, A. Lechner, A. M. Gigler, R. W. Stark, and W. Bensch, J. Micromech. Microeng. 21, 025001 (2011).

[7] C. Denniston and M. O. Robbins, J. Chem. Phys. 125, 214102 (2006).

[8] D. Ameur and G. Galliro, Microfluid Nanofluid 15, 183 (2013).

[9] H. J. C. Berendsen, J. R. Grigera, and T. P. Straatsma, J. Phys. Chem. 91, 6269 (1987).

[10] W. L. Jorgensen, J. D. Madura, and C. J. Swenson, J. Am. Chem. Soc. 106, 6638 (1984).

[11] W. L. Jorgensen, J. Phys. Chem. 90, 1276 (1986).

[12] D. Surblys, Y. Yamaguchi, K. Kuroda, M. Kagawa, T. Nakajima, and H. Fujimura, J. Chem. Phys. 140, 034505 (2014).

[13] M. P. Allen and D. J. Tildesley, Computer Simulation of Liquids (Clarendon Press, New York, 1989).

[14] G. Guevara-Carrion, J. Vrabec, and H. Hasse, J. Chem. Phys. 134, 074508 (2011).

[15] M. Lupkowski and F. van Swol, J. Chem. Phys. 93, 737 (1990).

[16] J. Blomer and A. Beylich, Surf. Sci. 423, 127 (1999).

[17] I. P. Omelyan, Comput. Phys. 12, 97 (1998).
[18] N. S. Martys and R. D. Mountain, Phys. Rev. E 59, 3733 (1999).

[19] L. Bocquet and J.-L. Barrat, Soft Matter 3, 685 (2007).

[20] K. Falk, F. Sedlmeier, L. Joly, R. R. Netz, and L. Bocquet, Nano Lett. 10, 4067 (2010).

[21] K. Falk, F. Sedlmeier, L. Joly, R. R. Netz, and L. Bocquet, Langmuir 28, 14261 (2012).

[22] R. J. Hardy, J. Chem. Phys. 76, 622 (1982).

[23] J.-G. Weng, S. Park, J. R. Lukes, and C.-L. Tien, J. Chem. Phys. 113, 5917 (2000).

[24] M. Dizechi and E. Marschall, J. Chem. Eng. Data 27, 358 (1982).

[25] R. Kubo, J. Phys. Soc. Jpn. 12, 570 (1957).

[26] D. M. Huang, C. Sendner, D. Horinek, R. R. Netz, and L. Bocquet, Phys. Rev. Lett. 101, 226101 (2008).

[27] T. Young, Phil. Trans. R. Soc. Lond. 95, 65 (1805).

[28] L. Bocquet and J.-L. Barrat, Phys. Rev. E 49, 3079 (1994).

[29] L. Bocquet and J.-L. Barrat, J. Chem. Phys. 139, 044704 (2013).

[30] S. Nakaoka, D. Surblys, Y. Yamaguchi, K. Kuroda, T. Nakajima, and H. Fujimura, Chem. Phys. Lett. 591, 306 (2014).

[31] D. Surblys, Y. Yamaguchi, K. Kuroda, T. Nakajima, and H. Fujimura, J. Chem. Phys. 135, 014703 (2011).

[32] R. F. Probstein, Physicochemical Hydrodynamics (WileyInterscience, New York, 1994).

[33] E. Helfand, Phys. Rev. 119, 1 (1960).

[34] B. Hess, J. Chem. Phys. 116, 209 (2002).

[35] H. C. Andersen, J. Chem. Phys. 72, 2384 (1980). 